\title{
Modificação da técnica de abordagem ventral à articulação atlantoxial sem a secção do músculo esternotireóideo
}

\author{
Modification of ventral technique approach to the articulation atlantoxial with no section of the muscle \\ sternothyreoid
}

\author{
Rafael Festugatto $^{\text {I* }}$ Alexandre MazzantiII Alceu Gaspar RaiserII Charles Pelizzari ${ }^{I}$ \\ Diego Vilibaldo Beckmann ${ }^{\mathrm{I}}$ Fernanda Souza Barbosa da Silva ${ }^{\mathrm{I}}$ Rogério Rodrigues Santos ${ }^{\mathrm{I}}$ \\ Dakir Polidoro III
}

\section{RESUMO}

O objetivo deste trabalho foi apresentar uma variação na técnica de acesso ventral à articulação atlantoaxial para tratamento da instabilidade atlantoaxial sem a secção do músculo esternotireóideo. Foram utilizados 15 cães, pesando entre oito e $12 \mathrm{~kg}$, sem raça definida, independente do sexo, distribuídos aleatoriamente em três grupos iguais de acordo com o período pós-operatório (PO) denominados de I (30dias), II (60 dias) e III (90 dias) para avaliações clínicas diárias. A articulação atlantoaxial foi submetida à artrodese por meio do acesso ventral utilizando pinos de Steinmann associados à resina acrílica autopolimerizável. O acesso e a exposição da articulação atlantoaxial sem a secção do músculo esternotireóideo foram realizados sem complicações ou limitações adicionais. Nenhum cão desta pesquisa apresentou tosse, dispnéia, regurgitação, paralisia laríngea ou Síndrome de Horner. Pode-se concluir que a secção do músculo esternotireóideo é um procedimento desnecessário e que não interfere na exposição da articulação atlantoaxial e na realização da artrodese em cães por meio do acesso ventral.
\end{abstract}

- NOTA-
Palavras-chave: artrodese atlantoaxial, neurologia, cão.

\section{ABSTRACT}

The aim of this research was to present a variation of the ventral technique access to the atlantoaxial joint, for treatment of atlantoaxial instability with no section of sternothyreoid muscle. Fifteen dogs, with weight between 8 and $12 \mathrm{~kg}$, were randomly distributed in three groups denominated one (30 days), two (60 days) and three (90 days) for daily clinical evaluations. The atlantoaxial joint was submitted to the arthrodesis through the ventral access using pins of Steinmann associated to acrylic resin. The access and exhibition of the atlantoaxial joint with no section of the sternothyreoid muscle was obtained without complications or additional limitations. No dog of this research presented coughing, dyspnea, regurgitation, laryngeal paralysis or Horner's syndrome. It can be concluded that the section of sternothyreoid muscle is an unnecessary procedure and that there is is no interference in the exhibition of atlantoaxial joint and arthrodesis approach of dogs through ventral access.

Key words: atlantoaxial arthrodesis, sternothyreoid, neurology, dog.

A instabilidade atlantoaxial (IAA) é uma afecção que pode resultar na compressão da medula espinhal (MOREAU, 2000) e conseqüentemente no surgimento de deficiências neurológicas. As causas podem ser traumáticas ou congênitas, sendo a ausência e a hipoplasia do processo odontóide do áxis as mais encontradas em cães (THACHER, 1996).

Os casos clínicos de instabilidade atlantoaxial geralmente resultam de uma combinação de fatores congênitos e traumáticos. Um animal com ausência congênita de suporte para o processo odontóide sofre um enfraquecimento progressivo do ligamento atlanto-

\footnotetext{
'Programa de Pós-graduação em Medicina Veterinária (PPGMV), Centro de Ciências Rurais (CCR), Universidade Federal de Santa Maria (UFSM). Avenida Roraima, n. 1000, Bairro Camobi, 97105-900, Santa Maria, RS, Brasil. E-mail: rfestuga@yahoo.com.br. *Autor para correspondência.

IIDepartamento de Clínica de Pequenos Animais, CCR, UFSM, Santa Maria, RS, Brasil.

IIICurso de Medicina Veterinária, CCR, UFSM, Santa Maria, RS, Brasil.
} 
axial dorsal. Finalmente, um traumatismo pequeno romperá o ligamento e precipitará a instabilidade (SHIRES, 1996; STAINKI et al., 1999). As raças toy, especialmente Poodles, Yorkshire Terriers, Teckel Miniatura, Chihuahuas e Spitz Alemão Anão, com menos de um ano de idade são freqüentemente as mais acometidas (BEAVER et al., 2000).

Conforme SHARP \& WHEELER (2005), os sinais neurológicos refletem a compressão da medula espinhal e são variáveis. Os pacientes geralmente possuem dor cervical, e a flexão ventral da cabeça freqüentemente exacerbará a dor e poderá piorar a condição neurológica (WAGNER, 1998; SEIM, 2002).

A IAA em cães pode ser tratada por meios conservadores ou cirúrgicos. O tratamento conservador é proposto para animais com sinais clínicos mínimos, já o tratamento cirúrgico é indicado para aqueles que apresentam deficiências neurológicas moderadas a graves (BAGLEY, 1999). O tratamento cirúrgico inclui técnicas de estabilização dorsal e ventral da articulação atlantoaxial (SCHULZ et al., 1997). Os objetivos dessas cirurgias são a redução da instabilidade articular e a descompressão da medula espinhal e das raízes nervosas (SEIM, 2002).

Dentre as técnicas empregadas, a artrodese atlantoaxial mediante aplicação ventral de dois pinos de Steinmann associados à resina acrílica autopolimerizável demonstrou melhores resultados (KNIPE et al., 2002). A técnica cirúrgica pelo acesso ventral inclui a incisão de pele e tecido subcutâneo seguida pela exposição dos músculos esternoiódeos, que são afastados para visualização da traquéia. $\mathrm{O}$ músculo esternotireóideo (MET) é mobilizado e seccionado próximo à sua inserção na laringe. Após o procedimento de imobilização da AA, é necessária a síntese do MET, além dos outros planos teciduais (SHARP \& WHEELER, 2005).

O músculo esternotireóideo dobra lateralmente na parte cranial do pescoço para inserirse na superfície lateral da lâmina tireóide da laringe, logo caudal à origem do músculo tireoióideo. Ele possui uma intersecção fibrosa transversa onde se separa do músculo esternoióideo. Esses músculos afastam a laringe, o aparelho hióide e a língua caudalmente (GETTY, 1986).

A literatura consultada apenas descreve a técnica cirúrgica por acesso ventral à articulação atlantoaxial (AA), mas não cita a importância e a necessidade da secção do músculo esternotireóideo durante o procedimento cirúrgico. O objetivo deste trabalho é apresentar uma variação na técnica de acesso ventral à AA sem a secção do MET. O intuito dessa modificação é evitar um trauma desnecessário provocado pela miotomia e suas possíveis conseqüências indesejáveis no período trans e pósoperatório e, em alguns casos, pela dificuldade na realização da miorrafia desse músculo. Para isso, foram utilizados 15 cães, independente do sexo, sem raça definida, pesando entre oito e $12 \mathrm{~kg}$, obtidos no Biotério Central da Universidade Federal de Santa Maria.

Os 15 cães foram distribuídos, aleatoriamente, em três grupos de igual número de acordo com o período pós-operatório (PO) denominados de grupos I, em que foram avaliados por 30 dias, II, 60 dias e III, 90 dias de PO. Todos os cães foram submetidos à artrodese atlantoaxial mediante 0 uso de pinos de Steimann associados à resina acrílica autopolimerizável.

Após jejum hídrico e alimentar de 12 horas, os animais foram tranqüilizados com $0,1 \mathrm{mg} \mathrm{kg}^{-1}$ de acepromazina $^{\mathrm{a}} \mathrm{IV}$, submetidos à anestesia geral com $4 \mathrm{mg} \mathrm{kg}^{-1}$ de propofolb $\mathrm{IV}$, intubados com sonda endotraqueal de diâmetro adequado e mantidos sob anestesia inalatória com halotanoc diluído em 100\% de $\mathrm{O}_{2}$, em circuito circular valvular. Foi administrado antibiótico profilático com ampicilina sódica ${ }^{\mathrm{d}}\left(30 \mathrm{mg} \mathrm{kg}^{-1}\right.$ ) intravenosa 30 minutos antes do início da cirurgia.

Depois de realizada a antissepsia do campo operatório com álcool-iodo-álcool e com o animal posicionado em decúbito dorsal com um anteparo de apoio sob o pescoço, foi realizada a artrodese atlantoaxial empregando dois pinos de Steimann associados à resina acrílica autopolimerizável, conforme técnica descrita por (SHARP \& WHEELER, 2005), mas sem realizar a secção do MET. Os animais foram submetidos à terapia antiinflamatória de cetoprofeno $10 \%^{\mathrm{e}}\left(2,0 \mathrm{mg} \mathrm{kg}^{-1}\right.$ de peso corporal, IM, por três dias) e sulfato de morfina $\left(0,3 \mathrm{mg} \mathrm{kg}^{-1}\right.$, via subcutânea, 6/6 horas, por 24 horas). Para isso, foi realizada uma incisão longitudinal mediana de pele e tecido subcutâneo que se estendeu cranialmente à laringe. Em seguida, incisouse a fáscia superficial expondo-se os músculos esternoióideos, que foram afastados para exposição da traquéia por meio de dissecação romba. O músculo esternotireóideo, a traquéia, a artéria carótida, a veia jugular e o tronco vagossimpático foram deslocados lateralmente (Figura 1), protegidos por compressas umedecidas em solução fisiológica estéril e mantidos com afastador auto-estático de Gosset. A fáscia profunda foi seccionada para exposição dos músculos longos do pescoço. Utilizando-se um elevador de periósteo, foram rebatidos os tendões desses músculos inseridos no processo ventral de C1. Os ventres musculares foram elevados caudolateralmente desde o corpo de C2. Dissecou-se a fáscia, e incisou-se a cápsula articular para exposição da cartilagem com 


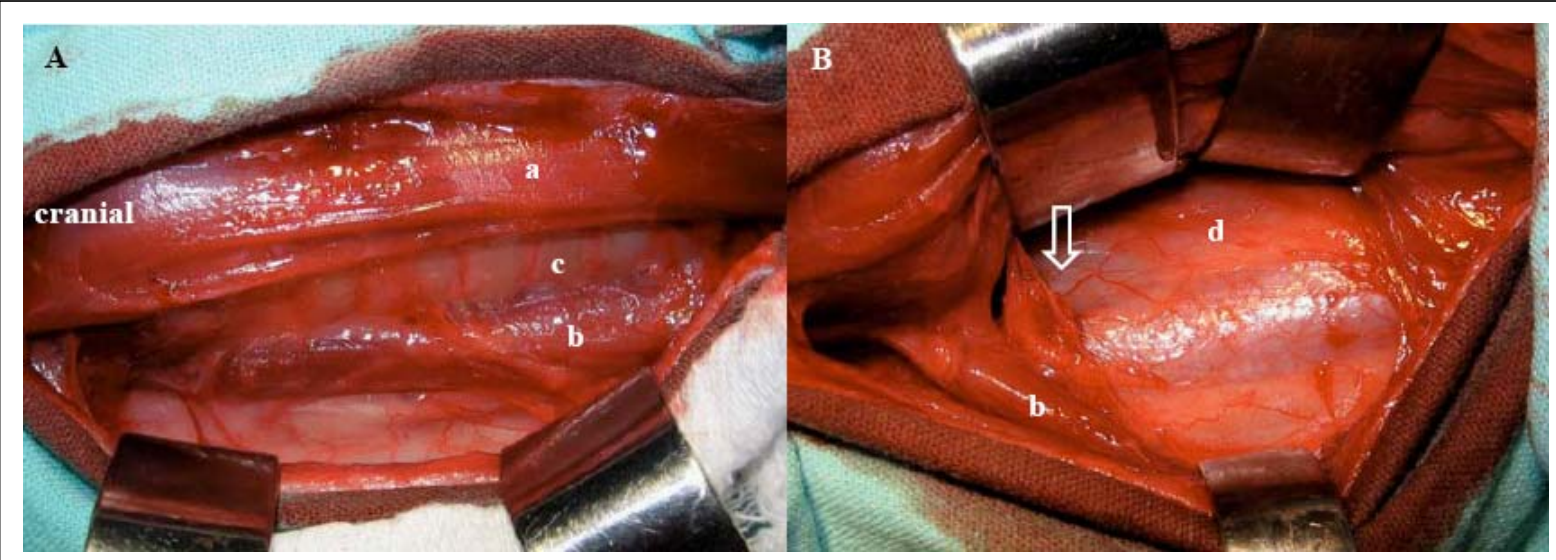

Figura 1 - Modificação da técnica de abordagem ventral à articulação atlantoxial sem a secção do músculo esternotireóideo. Incisão longitudinal mediana de pele e tecido subcutâneo cranial à laringe. A - Músculo esternoióideo (a), esternotireóideo direito (metd) (b) e traquéia (c) expostos. B - Metd retraído lateralmente permitindo visualização dos músculos longos do pescoço (d) para exposição da articulação atlantoaxial ( $\Downarrow$ ).

auxílio de um extrator de Hohmann. Em seguida, foi realizada a remoção da cartilagem articular por meio de uma broca sulcada de $4 \mathrm{~mm}$ acoplada a uma perfuratriz elétrica de baixa rotação. Para fixação da articulação atlanto-axial, foi utilizado pino de Steinmann de 1,0mm acoplado a uma parafusadeira de baixa rotação. O implante foi introduzido na extremidade cranial de $\mathrm{C} 2 \mathrm{a}$ $1,5 \mathrm{~cm}$ da borda e numa angulação aproximada de $30^{\circ}$ em relação ao eixo longitudinal da vértebra em direção à C1. O mesmo procedimento de fixação foi realizado com um segundo pino.

Todos os animais foram submetidos a avaliações clínicas diárias para detecção de possíveis complicações, tais como presença de tosse, dispnéia, regurgitação, paralisia laríngea, alteração na marcha, edema na região ventral do pescoço e Síndrome de Horner. Os resultados foram anotados em protocolos específicos.

O acesso e a exposição da articulação atlantoaxial (AA) sem a secção do músculo esternotireóideo foram realizados sem complicações ou limitações adicionais. Depois de exposta, a AA pôde ser manipulada de maneira satisfatória, possibilitando a realização dos procedimentos que envolvem a sua imobilização, discordando de SHARP \& WHEELER (2005), os quais afirmam que a secção desse músculo é útil.

A não realização da secção do MET elimina o risco de ruptura do feixe vascular que se localiza próximo ao local previsto para a miotomia. Esse vaso supre a glândula tireóide e deve ser preservado (SHARP \& WHEELER, 2005). No entanto, essa preservação nem sempre é possível. Além de manter sua estrutura anatômica fisiológica normal, ao preservar o MET, evita-se a hemorragia inerente ao rompimento das fibras musculares e as possíveis aderências aos tecidos adjacentes proporcionadas pela colocação de fios de sutura necessários à miorrafia.

SHORES \& TEPPER (2007) revisaram os registros médicos de cinco cães submetidos à estabilização da articulação atlantoaxial. Foi utilizado nesses animais um acesso ventral parasagital que separa os músculos esternocefálico e esternotireóideo direito. Ao contrário, neste trabalho foi realizada a separação dos músculos esternoióideos pareados e foi mantida a ligação entre os músculos separados por aqueles autores. A boa exposição da articulação atlantoaxial proporcionada pelo acesso parasagital, destacada pelos autores, também foi alcançada com o procedimento cirúrgico empregado nos animais do presente estudo.

Nenhum cão desta pesquisa apresentou tosse, dispnéia, regurgitação, paralisia laríngea ou Síndrome de Horner, embora BEAVER et al. (2000) tenha citado que a traquéia e os tecidos moles adjacentes podem ser traumatizados durante a retração lateral. SANDERS et al. (2004) relataram a ocorrência de um caso de paralisia laríngea e outros dois de dispnéia, um destes com Síndrome de Horner concomitante em 12 cães nos quais foi utilizada a técnica ventral de acesso à articulação atlantoaxial. Dificuldade respiratória e Síndrome de Horner foram também citadas por SCHULZ et al. (1997) como complicações pós-operatórias em dois de nove cães que receberam a aplicação ventral de pinos e resina acrílica para tratamento da instabilidade atlantoaxial. 
PLATT et al.(2004) realizaram um estudo retrospectivo de 19 cães com subluxação atlantoaxial tratados cirurgicamente por um método modificado de estabilização ventral com utilização de fio ortopédico e parafusos corticais associados à resina acrílica. $\mathrm{O}$ acesso à articulação foi realizado por meio da técnica descrita por SHARP \& WHEELER (2005). Embora não tenham encontrado nenhuma complicação intraoperatória, os autores descreveram o aparecimento de dois casos de pneumonia por aspiração documentados dentro de 24 horas após a cirurgia.

Em cinco cães deste experimento, foi encontrado edema na face ventral do pescoço ao redor da incisão cirúrgica, mas que regrediu até o sétimo dia de PO. Isso provavelmente tenha ocorrido pela manipulação dos tecidos e pela localização cirúrgica que favorecem o acúmulo de líquidos no espaço intersticial. De acordo com os resultados obtidos no presente experimento, pode-se concluir que a secção do músculo esternotireóideo é um procedimento desnecessário e que não interfere na exposição da AA e na realização da artrodese em cães por meio do acesso ventral.

\section{FONTES DE AQUISIÇÃO}

a-Acepran 0,2\%, Univet S.A., São Paulo - SP. b-Propofol-Lipuro 1\% - infarmed.cnf@infarmed.pt c-Tanohalo, Cristália Prod. Farm. Ltda, Itapira - SP. d-Ampicilina Sódica - Ampicillin/União Química - SP. e-Cetoprofeno $10 \%$ - Ketofen/Merial - SP.

\section{REFERÊNCIAS}

BAGLEY, R.S. Medula espinhal e vértebras. In: HARARI, J. Cirurgia de pequenos animais. Porto Alegre: Artes Médicas, 1999. Cap.22, p.347-358.

BEAVER, P. et al. Risk factors affecting the outcome of surgery for atlantoaxial subluxation in dogs: 46 cases (1978-1998). Journal of the American Veterinary Medical Association, v.216, n.7, p.1104-1109, 2000.

GETTY, R. Anatomia dos animais domésticos-Sisson/ Grossman. 5.ed. Rio de Janeiro: Guanabara Koogan, 1986. V.2, p.1421-1423.
KNIPE, M.F., et al. Atlantoaxial instability in 17 dogs. Journal Veterinary Internal Medicine, v.16, p.368-370, 2002.

MOREAU, P. Inestabilidad vertebral. In: PELLEGRINO, F. Et al. Síndromes neurológicos em perros y gatos. Buenos Aires: Panamericana, 2000. Cap.7, p.161-171.

PLATT, R.S. et al. A modified ventral fixation for surgical management of atlantoaxial subluxation in 19 dogs. Veterinary Surgery, v.33, p.349-354, 2004. Disponível em: <http:// www3.interscience.wiley.com/journal/118771329/ abstract?CRETRY $=1 \&$ SRETRY $=0>$. Doi: $10.1111 / \mathrm{j} .1532-$ 950X.2004.04050.x.

SANDERS, G. et al. Outcomes and complications associated with ventral screws, pins, and polymethylmethacrylate for atlantoaxial instability in 12 dogs. Journal of the American Animal Hospital Association, v.43, p.204-210, 2004.

SCHULZ, K.S. et al. Application of ventral pins and polymethylmethacrylate for the management of atlantoaxial instability: Results in nine dogs. Veterinary Surgery, v.26, n.4, p.317-325, 1997. Disponível em: <http:// www3.interscience.wiley.com/journal/119831161/abstract>. Doi: 10.1111/j.1532-950X.1997.tb01504.x.

SEIM, H.S. Cirurgia da espinha cervical. In: FOSSUM, T.W. Cirurgia de pequenos animais. São Paulo: Roca, 2002. Cap.34, p.1157-1215.

SHARP, N.J.H.; WHEELER, S.J. Small animal spinal disorders. diagnosis and surgery. 2.ed. Philadelphia: Elsevier Mosby, 2005. 380p.

SHIRES, P.K. Instabilidade atlantoaxial. In: BOJRAB, M.J. Et al. Técnicas atuais em cirurgia de pequenos animais. 3.ed. São Paulo: Roca, 1996. Cap.42, p.545-564.

SHORES, A.; TEPPER, L.C. A modified ventral approach to the atlantoaxial junction in the dog. Veterinary Surgery, v.36, p.765-770, 2007.

STAINKI, D.R. Et al. Instabilidade atlanto-axial em canino: breve revisão e relato de caso. Revista Faculdade Zootecnia Veterinária Agronomia, v.5/6, n.1, p.77-81, 1998/99.

THACHER, C. Biomecânica das fraturas cranianas, espinais e luxações. In: BOJRAB, M.J. Mecanismos da moléstia na cirurgia de pequenos animais. 2.ed. São Paulo: Manole, 1996. Cap.135, p.1150-1160.

WAGNER, S.D. Fratura e deslocamento espinhais. In: BIRCHARD, S.J.; SHERDING, R.G. Manual Saunders - Clínica de pequenos animais. 4.ed. São Paulo: Roca, 1998. Cap.5, p1081-1090. 